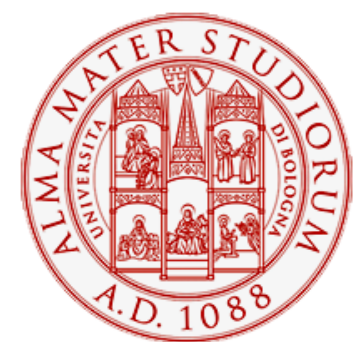

Alma Mater Studiorum - Università di Bologna DEPARTMENT OF ECONOMICS

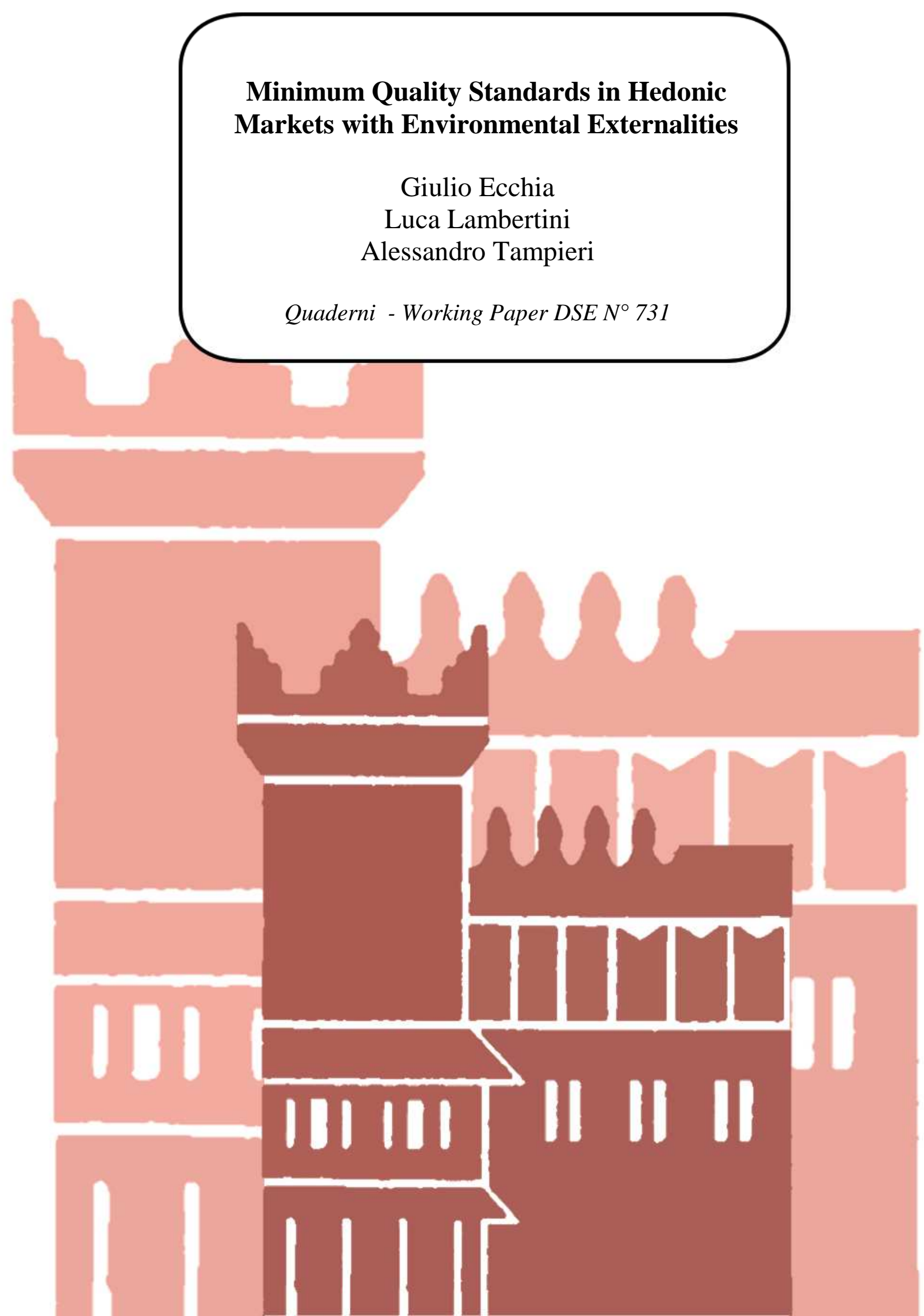




\title{
Minimum Quality Standards in Hedonic Markets with Environmental Externalities*
}

\author{
Giulio Ecchiał, Luca Lambertini; Alessandro Tampieri ${ }^{\S}$ \\ University of Bologna
}

February 4, 2011

\begin{abstract}
We investigate the introduction of a minimum quality standard (MQS) in a vertically differentiated duopoly with an environmental externality. We establish that the MQS bites only if the hedonic component of consumer preferences is sufficiently strong. Then, we illustrate an underlying tradeoff between the beneficial effects of quality enhancement on prices and the associated undesirable increase in the environmental externality.
\end{abstract}

JEL Numbers: L13, L51, Q50.

Keywords: MQS, environmental externality, product quality.

*We would like to thank HERA spa for sponsoring this project. The usual disclaimer applies. Italy

${ }^{\dagger}$ Department of Economics, University of Bologna, Piazza Scaravilli 2, 40126 Bologna,

${ }^{\ddagger}$ Department of Economics, University of Bologna, Strada Maggiore 45, 40125, Bologna, Italy; email: luca.lambertini@unibo.it.

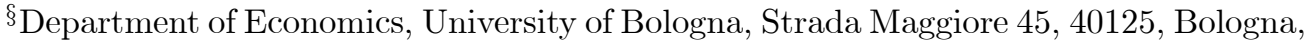
Italy; email: alessandro.tampieri@unibo.it. 


\section{Introduction}

So far, the relatively small literature on minimum quality standards (MQSs) in vertically differentiated industries has focussed on use of MQS regulation to correct the downward distortion of product quality due to market power (see Ronnen, 1991; Crampes and Hollander, 1995; Ecchia and Lambertini, 1997, inter alia). Few efforts have been carried out to investigate the optimal design of an MQS and its consequences in markets where production entails a negative environmental externality. In this vein, Arora and Gangopadhyay (1995), Lutz et al. (2000) and André et al. (2009) investigate the role of MQS in models where quality has a definite environmental impact. The same feature can be found also in Lombardini-Riipinen (2005), where regulation is carried out via taxation on polluting emissions.

What we do in this note is instead to assess MQS regulation in a duopoly model where quality is a purely hedonic variable, consumers are myopic and pollution is proportional to the industry output. Accordingly, quality affects the externality only indirectly, via the interplay with equilibrium prices and outputs, but has no intrinsic 'green' features. This can be the case in industries like consumer electronics, where standards are directed, e.g., to improve the quality of LCDs for computers and TV sets without accounting for the environmental implications of large scale production.

Our results can be summarised as follows. To begin with, the MQS bites only if the hedonic component of consumer preferences is high enough compared to the marginal environmental damage associated with production. In such a case, the adoption of a binding MQS that diminishes product differentiation and increases industry output poses a tradeoff between the price effect and the external effect, as consumers are able to purchase larger quantities at a lower price and enjoy a higher average quality, but this goes along with a higher amount of pollution. Hence, a binding MQS improves welfare not by shrinking the environmental damage, but simply by increasing the average quality supplied to the market. 


\section{The model}

We consider a duopoly market for vertically differentiated products supplied by single-product firms. The demand side is modelled à la Mussa and Rosen (1978). There is a continuum of consumers whose types are identified by $\theta$, uniformly distributed with density equal to one in the interval $[0, \Theta]$. Parameter $\theta$ represents the consumers' marginal willingness to pay for quality. Each consumer is assumed to buy at most one unit of the vertically differentiated good in order to maximise the following surplus function:

$$
U=\theta q_{i}-p_{i}
$$

where $q_{i} \in[0, Q]$ indicates the quality of the product and $p_{i}$ is the market price at which that variety is supplied by firm $i=H, L$, with $q_{H} \geq q_{L}$. Therefore, the consumer who is indifferent between $q_{H}$ and $q_{L}$ is identified by the level of marginal willingness to pay $\widehat{\theta}$ that solves

$$
\widehat{\theta} q_{H}-p_{H}=\widehat{\theta} q_{L}-p_{L}
$$

and therefore $\widehat{\theta}=\left(p_{H}-p_{L}\right) /\left(q_{H}-q_{L}\right)$. Thus, market demand for the highquality good is $x_{H}=\Theta-\widehat{\theta}$. We assume partial market coverage, so that there is another consumer, identified by $\widetilde{\theta}$, who is indifferent between buying $q_{L}$ or not buying at all:

$$
\widetilde{\theta} q_{L}-p_{L}=0
$$

whereby $\widetilde{\theta}=p_{L} / q_{L}$ and the demand for the inferior variety is $\widehat{\theta}-\widetilde{\theta}$. Accordingly, we can define consumer surplus as follows:

$$
C S=\int_{\widetilde{\theta}}^{\widehat{\theta}}\left(k q_{L}-p_{L}\right) d k+\int_{\widehat{\theta}}^{\Theta}\left(z q_{H}-p_{H}\right) d z .
$$

On the supply side, for the sake of simplicity, we normalise their produc- 
tion costs to zero, so that profit functions are $\pi_{H}=p_{H} x_{H}$ and $\pi_{L}=p_{L} x_{L} \cdot{ }^{1}$

Production goes along with a negative environmental externality $s=$ $b\left(x_{H}+x_{L}\right)$, with $b>0$. Note that the good has the same environmental impact irrespective of its quality. Also, note that consumers are assumed to be myopic, in the sense that (1) does not account for the presence of pollution. ${ }^{2}$ Social welfare is determined by the sum of profits and consumer surplus, minus the environmental externality:

$$
W=C S+\pi_{H}+\pi_{L}-s .
$$

Competition takes place in two stages. In the first, firms choose qualities and in the second they compete in prices. The solution concept is the subgame perfect equilibrium by backward induction.

\section{Results}

To begin with, we characterise optimal prices for any given quality pair. These are the same as in Choi and Shin (1992):

$$
p_{H}^{N}=\frac{2 m q_{H}\left(q_{H}-q_{L}\right)}{4 q_{H}-q_{L}} ; p_{L}^{N}=\frac{m q_{L}\left(q_{H}-q_{L}\right)}{4 q_{H}-q_{L}}
$$

where superscript $N$ stands for Nash equilibrium.

As a preliminary result, note that, given the equilibrium prices, the introduction of an MQS may have ambiguous effects. Indeed, by substituting

\footnotetext{
${ }^{1}$ Quality can be thought of as the result of R\&D efforts previously carried out by firms and summarised in a fixed $\operatorname{cost} \varepsilon>0$ that is taken to be small enough for profits to be positive throughout.

${ }^{2}$ It is worth noting that this is not a crucial assumption, as admitting the possibility for consumers to be environmentally concerned, with $U=\theta q_{i}-p_{i}-s$ would not modify the expressions of $\widehat{\theta}$ and $\widetilde{\theta}$ resulting from $\widehat{\theta} q_{H}-p_{H}-s=\widehat{\theta} q_{L}-p_{L}-s$ and $\widetilde{\theta} q_{L}-p_{L}-s=-s$, respectively.
} 
the equilibrium prices in $s$, we obtain:

$$
s=\frac{3 b m q_{L}}{4 q_{H}-q_{L}}
$$

Differentiation with respect to $q_{L}$ yields

$$
\frac{\partial s}{\partial q_{L}}=\frac{3 b m q_{H}}{\left(4 q_{H}-q_{L}\right)^{2}}>0 .
$$

As a consequence, an increase in $q_{L}$, like that generated by an MQS, brings about an increase in pollution parallel to an increase in industry output, as $\partial s / \partial q_{L} \propto \partial\left(x_{H}+x_{L}\right) / \partial q_{L}$. This implies:

Lemma 1 There exists a tradeoff between the price effect and the external effect of MQS regulation, whereby a priori expanding output has ambiguous consequences.

We can move on to the first stage of the unregulated game, where the first order condition (FOC) $\partial \pi_{L} / \partial q_{L}=0$ yields the best reply $q_{L}^{*}=4 q_{H} / 7$, while $\partial \pi_{H} / \partial q_{H}>0$ always, so that $q_{H}^{N}=Q$ and $q_{L}^{N}=4 Q / 7$ at the unregulated subgame perfect equilibrium, as we know from Choi and Shin (1992). Individual firms' outputs are $x_{H}^{N}=7 \Theta / 12$ and $x_{L}^{N}=7 \Theta / 24$. The corresponding consumer surplus is $C S^{N}=7 \Theta^{2} Q / 24$, while profits amount to $\pi_{H}^{N}=7 \Theta^{2} Q / 48$ and $\pi_{L}^{N}=\Theta^{2} Q / 48$. Pollution and social welfare are $s^{N}=7 b \Theta / 8$ and $W^{N}=\Theta(11 \Theta Q-21 b) / 24$, respectively, with $W^{N}>0$ for all $\Theta Q>21 b / 11$. This establishes that the hedonic effect driving consumer surplus must outweigh the external effect.

In the regulated case, the government introduces an MQS affecting directly the behaviour of firm $L$. We reconstruct the introduction of the MQS via simultaneous play between firm $H$ and the regulator at the first stage. Firm $H$ 's FOC remains unchanged, while the regulator solves:

$$
\frac{\partial W}{\partial q_{L}}=\frac{\Theta Q\left(\Theta Q\left(20 Q-17 q_{L}\right)-6 b\left(4 Q-q_{L}\right)\right.}{2\left(4 Q-q_{L}\right)^{3}}=0
$$


which is always positive if $b=0$, i.e., in the limit case where pollution is absent altogether. From (9) we obtain:

$$
q_{L}^{M Q S}=\frac{4 Q(5 \Theta Q-6 b)}{17 \Theta Q-6 b} \in(0, Q) \forall \Theta Q \in\left(\frac{6 b}{5}, 6 b\right)
$$

Note that (10) entails that vertical differentiation disappears through the adoption of the MQS whenever the weight of pollution is low enough, i.e., for all $b \in[0, \Theta Q / 6]$. Moreover, from (10) one gets:

$$
\frac{\partial q_{L}^{M Q S}}{\partial \Theta}=\frac{288 \Theta Q^{2}}{(17 \Theta Q-6 b)^{2}}=-\frac{\partial q_{L}^{M Q S}}{\partial b} .
$$

Accordingly, we may state:

Lemma 2 The $M Q S$ decreases in $b$ and increases in $\Theta$ for all $\Theta Q \in(6 b / 5,6 b)$. For all $\Theta Q \geq 6 b$, the $M Q S$ is constant at $Q$.

The intuitive reason is that, since the MQS expands industry output, $q_{L}^{M Q S}$ reacts positively to an increase in market affluency and negatively to an increase in the weight of the external effect. This applies if the hedonic dimension of the market is outweighted by the external effect. Otherwise, the design of the MQS is driven solely by the hedonic dimension, leading the industry towards the replication of the perfectly competitive outcome. In the remainder, we focus on the parameter range wherein some degree of product differentiation exists.

At the regulated equilibrium, consumer surplus is:

$$
C S^{M Q S}=\frac{(7 \Theta Q-6 b)(17 \Theta Q-6 b)}{192 Q},
$$

while the profits amount to:

$$
\pi_{H}^{M Q S}=\frac{(\Theta Q-6 b)(17 \Theta Q-6 b)}{192 Q} ; \pi_{L}^{M Q S}=\frac{(\Theta Q-6 b)(5 \Theta Q-6 b)}{192 Q},
$$


both positive for $\Theta Q \in(6 b / 5,6 b)$. Pollution is:

$$
s^{M Q S}=\frac{b(17 \Theta Q-6 b)}{16 Q},
$$

and thus social welfare is:

$$
W^{M Q S}=\frac{36 b^{2}-204 b \Theta Q+97 \Theta^{2} Q}{192 Q}>0 \forall \Theta Q>\frac{6(17+8 \sqrt{3})}{97} \simeq 1.908 b .
$$

Now observe that the MQS may not be binding, since

$$
\frac{4 Q(5 \Theta Q-6 b)}{17 \Theta Q-6 b}>\frac{4 Q}{7} \text { iff } \Theta Q>2 b
$$

that is, for all $\Theta Q \in(21 b / 11,2 b)$ the MQS is indeed a maximum quality standard.

Provided $\Theta Q>2 b$ (so that $q_{L}^{M Q S}$ bites), it is also easy to verify that $x_{i}^{M Q S}>x_{i}^{N}, i=H, L$, and therefore total industry output indeed increases after the adoption of a binding MQS, because the latter brings about tougher price competition which translates into a demand increase for both varieties. Exactly the opposite applies for $\Theta Q \in(21 b / 11,2 b)$.

It turns out that social welfare fully reflects this ambiguity:

Proposition 1 Take $\Theta Q \in(2 b, 6 b)$, so that some degree of vertical differentiation survives the introduction of a binding MQS. In such a range, $W^{M Q S}>W^{N}$ because the increase in consumer surplus outweighs the decrease in profits and the increase in pollution.

Proof. For all $\Theta Q \in(2 b, 6 b)$,

$$
\begin{gathered}
\pi_{H}^{M Q S}+\pi_{L}^{M Q S}-\pi_{H}^{N}+\pi_{L}^{N} \equiv \Delta \Pi=\frac{(\Theta Q-2 b)(2 b-3 \Theta Q)}{Q}<0 \\
C S^{M Q S}-C S^{N} \equiv \Delta C S=\frac{3(\Theta Q-2 b)(7 \Theta Q-2 b)}{64 Q}>0
\end{gathered}
$$




$$
S^{M Q S}-S^{N} \equiv \Delta S=\frac{3 b(\Theta Q-2 b)}{16 Q}>0 .
$$

As a consequence, the fact that in the same range $W^{M Q S}>W^{N}$, or equivalently

$$
\Delta \Pi+\Delta C S-\Delta S>0
$$

is entirely due to the price effect enhancing consumer surplus largely enough to more than offset the reduction in industry profits and the increase in the environmental externality.

At first sight, this result may indeed look quite counterintuitive. However, it can be spelled out in the following terms. Whenever the hedonic component of preferences is strong enough to yield a binding MQS, the resulting welfare increase is to be entirely imputed to the standard correction of the downward quality distortion generated by price competition rather than to a reduction in pollution. That is, if the market is affluent enough, the satisfaction of myopic consumers is more relevant than the increase in the external effect that necessarily goes along with it.

\section{References}

[1] André, F.J., González, P. and Porteiro, N. 2009. Strategic quality competition and the Porter hypothesis. Journal of Environmental Economics and Management 57: 182-194.

[2] Arora, S. and Gangopadhyay, S. 1995. Toward a theoretical model of voluntary overcompliance. Journal of Economic Behavior and Organization 28: 289-309.

[3] Choi, C. J. and Shin, H. S. 1992. A comment on a model of vertical product differentiation. Journal of Industrial Economics 40: 229-231. 
[4] Crampes, C. and Hollander, A. 1995. Duopoly and quality standards. European Economic Review 39: 71-82.

[5] Ecchia, G. and Lambertini, L. 1997. Minimum quality standards and collusion. Journal of Industrial Economics 45: 101-113.

[6] Lombardini-Riipen, C. 2005. Optimal tax policy under environmental quality competition. Environmental and Resource Economics 32: 317336.

[7] Lutz, S., Lyon, T. P. and Maxwell, J. W. 2000. Quality leadership when regulatory standards are forthcoming. Journal of Industrial Economics 48: 331-348.

[8] Mussa, M. and Rosen, S. 1978. Monopoly and product quality. Journal of Economic Theory 18: 301-317.

[9] Ronnen, U. 1991. Minimum quality standards, fixed costs, and competition. RAND Journal of Economics 22: 490-504. 


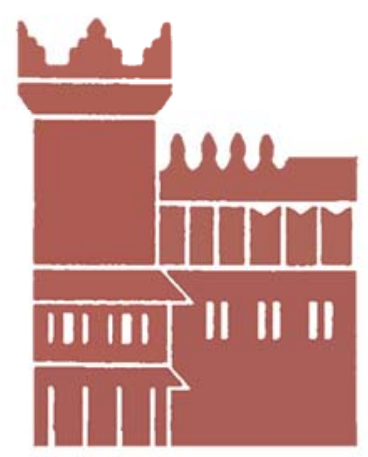

Alma Mater Studiorum - Università di Bologna DEPARTMENT OF ECONOMICS

Strada Maggiore 45

40125 Bologna - Italy

Tel. +39051 2092604

Fax +390512092664

http://www.dse.unibo.it 